\title{
Research Article \\ On Minimal Realizations and Minimal Partial Realizations of Linear Time-Invariant Systems Subject to Point Incommensurate Delays
}

\author{
M. De la Sen \\ Department of Electricity and Electronics, Institute for Research and Development of Processes, \\ Campus of Leioa (Bizkaia), Apartado 644, 48080 Bilbao, Spain
}

Correspondence should be addressed to M. De la Sen, manuel.delasen@ehu.es

Received 1 June 2007; Revised 22 November 2007; Accepted 28 November 2007

Recommended by Giuseppe Rega

\begin{abstract}
This paper investigates key aspects of realization and partial realization theories for linear timeinvariant systems being subject to a set of incommensurate internal and external point delays. The results are obtained based on the use of formal Laurent expansions whose coefficients are polynomial matrices of appropriate orders and which are also appropriately related to truncated and infinite block Hankel matrices. The above-mentioned polynomial matrices arise in a natural way from the transcendent equations associated with the delayed dynamics. The results are linked to the properties of controllability and observability of dynamic systems. Some related overview is given related to robustness concerned with keeping the realization properties under mismatching between a current transfer matrix and a nominal one.
\end{abstract}

Copyright ( $) 2008$ M. De la Sen. This is an open access article distributed under the Creative Commons Attribution License, which permits unrestricted use, distribution, and reproduction in any medium, provided the original work is properly cited.

\section{Introduction}

The realization theory is a very basic issuein theory of linear dynamic systems. It basically consists of a state-space realization from available external description as, for instance, a transfer matrix model or models based on measured sets of input-output data. The minimal realization problem of dynamic linear time-invariant delay-free systems is to find a linear statespace description of the minimal possible dimension whose associate transfer matrix exactly matches a proper predefined rational matrix with entries over a field. Any proper, that is, realizable, rational transfer matrix can be expanded in a formal Laurent series at infinity resulting in a formal identity of the Laurent series and the transfer matrix. The coefficients of the Laurent series form an infinite sequence of matrices which are the block matrices of the infinite block Hankel matrix. The minimal realization problem in the delay-free case may be 
focused on finding a state-space realization on minimal order, that is, as small as possible, so that the above-mentioned identity holds. The classical related problem was firstly formulated by Kalman $[1,2]$ for the single-input single-output case. The minimal partial realization problem of any approximation is formulated in terms of a certain finite-order truncation of the identity of the Laurent series expansion with the transfer matrix [3-7]. In this paper, this formalism is extended to linear time-invariant systems subject to any finite number of, in general, incommensurate internal (i.e., in the state) and external (i.e., in the input and/or output) point delays; namely, they are not all an integer multiple of a base delay, contrary to commensurate delays. These systems are very common in the real life, like, for instance, in biological problems, transportation problems, signal transmission, war/peace models, and so forth [8]. There is a wide recent interest in studying the properties of time-delay systems as associated to either linear dynamics or nonlinear dynamics or even to dynamics described by differential equations in partial derivatives [9-22]. Impulsive time-delay systems have been studied recently in $[9,12,16,19]$. In particular, impulses may be associated with the singularity of the dynamics and the loss of uniqueness of the solution as a result [16] or to the fact that the forcing terms are impulsive. The robust stability problem has been studied in [11] via linear matrix inequalities and Lyapunov's functions. The oscillatory behavior under delays and possible unmodeled dynamics is investigated in [10,21]. Different aspects and conditions of positivity of the solutions and equilibrium points have been recently described in [16-18, 22], either in the first orthant or in generic cones. The central purpose of this paper is concerned with the realization theory and associated properties of controllability and observability of dynamic systems under linear delayed dynamics. An infinite polynomial block Hankel matrix, as well as its associate $\tau$-finite polynomial block Hankel matrices, is defined in order to relate the spectral controllability and observability properties of minimal realizations $[3,4,8,23-31]$ with the minimum feasible finite rank of such a Hankel matrix. Then, such a rank is proved to equalize that of its associate finite polynomial block matrices whose orders exceed a minimum lower bound related to the minimal realization to be synthesized. Potential extensions of the obtained results might be addressed to investigate controllability and observability and then minimal and partial minimal realizations of expanded composite systems $[23,24]$ and hybrid systems $[25,31,32]$, including systems subject to switches between multiple parameterizations. Therefore, problems related to properties of dynamic systems like, for instance, controllability, observability, or realizability have received important attention from years up till now. An important point of view for modeling dynamic systems is to synthesize both nonparametric and parametric models which describe the mappings relating collections of measured input/output data as closely as possible. The Hankel matrix-based models are appropriate to describe linear input/output mappings by infinitely many parameters, in general, since they might be obtained directly from available input/output data on the system. In a second step, those models may be mapped either exactly or approximately into finitely parameterized models, for instance, via transfer matrices and associate state-space realizations $[1-4,6,7]$. In particular, the point of view of starting from Hankel matrices-based models formulated in rings, in general, has been discussed in $[3,4]$. Note that realizability results and related properties formulated over rings lead only to sufficiency-type conditions when applied to time-delay systems. The reason is that those systems are modeled by transfer matrices involving rational entries being quotients of quasipolynomials or their associate matrix impulse responses, infinite series, and so forth. It is known that bijective mappings exist between such quasipolynomials and their corresponding polynomials of several variables depending on the number of delays. However, 
quasipolynomials are in fact transcendent functions which depend only on one variable since there is a precise functional dependence on the remaining independent variables in the polynomial context and that one. Such a variable is, roughly speaking, the argument of the Laplace transform. In this paper, stronger results are obtained since the fact that only one independent variable exists is taken into account.

The paper is organized as follows. Section 2 deals with proper transfer matrix descriptions of linear time-invariant time-delay systems from their state-space realizations in the general case where delays are incommensurate. Section 3 establishes connections to the level of appropriate isomorphisms between formal Laurent expansions at infinity as well as their finite truncations of a certain finite order, rational transfer matrices, and rings of polynomials. It turns out that Laurent expansions at infinity are equivalent to transfer matrices while their truncations of a certain finite order are related to Hankel matrices. Such formalism is applied to linear time-invariant systems subject to internal incommensurate point delays. In particular, the properties of spectral controllability and observability are investigated together with the associated problems of minimal realizations for simultaneously controllable and observable systems and minimal partial state-space realizations. Related results are obtained for the independence of the delay case as well as for the dependence on the delay case. The related problems of synthesis of minimal state-space realizations and minimal partial realizations are dealt with in Section 4 with special emphasis on the single-input single-output case. The formulation is made in terms of finding a state-space realization such that it matches a certain transfer matrix which is formally identical to a series Laurent expansion at infinity. Finally, a section of concluding remarks ends the paper.

\subsection{Some basic state-space realization concepts and related notations}

The following concepts about state-space realizations,minimal realizations, and minimal partial realizations are dealt with through the manuscript.

(1) A proper rational transfer matrix $G(s)$ over a filed $\mathbf{K}$ takes values in $\mathbf{K}^{p \times m}(s)$, where $s$ is the Laplace transform variable.

(2) Any proper rational $G(s) \in \mathbf{K}^{p \times m}(s)$ can be expanded in a formal Laurent series at infinity leading to the formal identity $G(s)=\sum_{i=0}^{\infty} H_{i} s^{-i}$ with $\left\{H_{i}\right\}_{i \in \mathbb{N}_{0}}$ being an infinite sequence of matrices which are the block matrices of the infinite block Hankel matrix.

(3) The infinite block Hankel matrix is often denoted as $\left\{H_{i}\right\}_{0}^{\infty}$, with $\mathbb{N}_{0}=\mathbb{N} \cup\{0\}$ and $\mathbb{N}$ being the set of the natural numbers.

(4) The minimal realization problem in the delay-free system consists in finding astatespace realization $(A, B, C, D)$, with $A \in \mathbb{R}^{n \times n}, B \in \mathbb{R}^{n \times m}, C \in \mathbb{R}^{p \times n}$, and $D \in \mathbb{R}^{p \times m}$, of order $n \in \mathbb{N}$ being minimal, that is, as small as possible, so that, given the infinite sequence $\left\{H_{i} \in \mathbf{K}^{p \times m}\right\}_{0}^{\infty}$, the identity of the proper rational transfer matrix with the formal Laurent series at $\infty$ holds, that is, $G(s)=\sum_{i=0}^{\infty} H_{i} s^{-i}=C(s I-A)^{-1} B+D$ and then $H_{0}=D$ and $H_{i}=C A^{i-1} B, i \in \mathbb{N}$. A realization is minimal if and only if it is both controllable and observable.

(5) If $D=0$, then $G(s)$ is strictly proper; that is, the number of poles exceeds strictly the number of zeros at any entry of the transfer matrix. 
(6) The so-called minimal partial realization problem of any approximation $\tau$ is formulated as follows. Given a finite sequence $\left\{H_{i} \in \mathbf{K}^{p \times m}\right\}_{0}^{\tau}$ with some $\tau \in \mathbb{N}$, satisfying $H_{0}=D, H_{i}=C A^{i-1} B$, for all $i \in \bar{\tau}:=\{1,2, \ldots, \tau\}$, there exists a quadruple $(A, B, C, D)$, with $A \in \mathbb{R}^{n \times n}, B \in \mathbb{R}^{n \times m}, C \in \mathbb{R}^{p \times n}$, and $D \in \mathbb{R}^{p \times m}$, of minimal order $n \in \mathbb{N}$ (the order of $A$ ) such that $C(s I-A)^{-1} B+D=\sum_{i=0}^{\tau} H_{i} s^{-i}+0\left(s^{-\tau-1}\right)$.

\section{State-space and transfer matrix descriptions of time-delay systems}

Consider the linear time-invariant dynamic system in state-space form:

$$
\begin{aligned}
& \dot{x}(t)=\sum_{i=0}^{q} A_{i} x\left(t-h_{i}\right)+\sum_{i=0}^{q^{\prime}} B_{i} u\left(t-h_{i}^{\prime}\right), \\
& y(t)=C x(t)+D u(t),
\end{aligned}
$$

where $x: \mathbb{R}_{+} \times \Sigma \times U \rightarrow \Sigma \subset \mathbb{R}^{n}, u: \mathbb{R}_{+} \rightarrow U \subset \mathbb{R}^{m}, y: \mathbb{R}_{+} \times \Sigma \times U \rightarrow \mathrm{Y} \subset \mathbb{R}^{p}$ are the state, input, and output vector functions in their respective state, input, and output spaces $\Sigma, U$, and $Y$, where $\mathbb{R}_{+}:=\{t \in \mathbb{R}: t \geq 0\}, h_{0}=h_{0}^{\prime}=0$, and $h_{i}(>0) \in \mathbb{R}_{+}(i \in \bar{q}:=\{1,2, \ldots, q\})$, $h_{i}^{\prime}(>0) \in \mathbb{R}_{+}\left(i \in \bar{q}^{\prime}\right)$ are the internal and external point delays. If the input is generated via state feedback, then $u: \mathbb{R}_{+} \times \Sigma \rightarrow U \subset \mathbb{R}^{m}$. If it is generated via output feedback, then $u: \mathbb{R}_{+} \times Y \rightarrow U \subset \mathbb{R}^{m}$. The internal delays $\left(h_{i}\right)$, which are assumed to be pairwise distinct, and the external ones $\left(h_{j}^{\prime}\right)$, which are also assumed to be pairwise distinct, are both incommensurate delays; that is, they are not necessarily equal to $i h_{b}$ and $j h_{b}^{\prime}\left(i \in \bar{q}, j \in \bar{q}^{\prime}\right)$, with some $h_{b}>0$, $h_{b}^{\prime}>0 ; A_{i} \in \mathbb{R}^{n \times n}(i \in \bar{q} \cup\{0\}), B_{i} \in \mathbb{R}^{n \times m}\left(i \in \bar{q}^{\prime} \cup\{0\}\right), C \in \mathbb{R}^{p \times n}$, and $D \in \mathbb{R}^{p \times m}$ are matrices of real entries which parameterize (2.1)-(2.2). The dynamic system is subject to initial conditions, $\varphi:[-h, 0] \rightarrow \mathbb{R}^{n}$, where $h:=\operatorname{Max}_{i \in \bar{q}}\left(h_{i}\right)$ is piecewise continuous possibly with bounded discontinuities on a subset of zero measures of its definition domain. Closed formulas for the unique state and output trajectory solutions of (2.1)-(2.2) are provided in [33,34], although the dynamic system is infinite-dimensional by nature. One of these formulas is based on defining a $C_{0}$-semigroup generated by the infinitesimal generator of the delay-free matrix $A_{0}$-which is trivially valid for the time-invariant case only. An alternative second formula is based on an evolution operator which satisfies the unforced differential system (2.1) which may be generalized to the linear time-varying case. Both formulas are equivalent since the solutions are unique.

By taking right Laplace transforms in the state-space description (2.1)-(2.2) with $\varphi \equiv 0$, a transfer matrix exists, which is defined by

$$
G(s):=\frac{Y(s)}{U(s)}=\left[\frac{\operatorname{Lap}_{+}(y(t))}{\operatorname{Lap}_{+}(u(t))}\right]_{\varphi \equiv 0}=C\left(s I_{n}-\sum_{i=0}^{q} A_{i} e^{-h_{i} s}\right)^{-1}\left(\sum_{j=0}^{q^{\prime}} B_{i} e^{-h_{i}^{\prime} s}\right)+D,
$$

where $\operatorname{Lap}_{+}(v(t))$ is the right Laplace transform of $v: \mathbb{R}_{+} \rightarrow \mathbb{R}^{s}$ provided that it exists. Note that $G(s)$ is a complex matrix function in $C^{p \times m}$ in the complex indeterminate $s$ whose $(i, j)$ th entry is

$$
G_{i j}(s)=\frac{c_{i}^{T} \operatorname{Adj}\left(s I_{n}-\sum_{k=0}^{q} A_{i} e^{-h_{k} s}\right)\left(\sum_{j=0}^{q} b_{\ell j} e^{-h_{\ell}^{\prime} s}\right)}{\operatorname{Det}\left(s I_{n}-\sum_{k=0}^{q} A_{i} e^{-h_{k} s}\right)}+D_{i j}
$$


where $\operatorname{Adj}(\cdot)$ and $\operatorname{Det}(\cdot)$ stand for the adjoint matrix and the determinant of the $(\cdot)$-matrix, respectively, and $c_{i}^{T}$ and $b_{\ell j}$ are the $i$ th row of $C(i \in \bar{p})$ and the $j$ th column of $B_{\ell}\left(\ell \in \bar{q}^{\prime} \cup\{0\}\right)$, respectively. Define complex $\left(q+q^{\prime}\right)$ and $\left(\widehat{q}+\widehat{q}^{\prime}\right)$ tuples as follows:

$$
\begin{aligned}
& z:=\left(z^{I}, z^{E}\right)=\left(z_{1}, \ldots, z_{q}, z_{q+1}, z_{q+q^{\prime}}\right) \in \mathbf{C}^{q+q^{\prime}}, \\
& \widehat{z}:=\left(\hat{z}^{I}, \widehat{z}^{E}\right)=\left(z_{1}, \ldots, z_{\hat{q}}, z_{\hat{q}+1}, z_{\hat{q}+\hat{q}^{\prime}}\right) \in \mathbf{C}^{\hat{q}+\widehat{q}^{\prime}},
\end{aligned}
$$

where

$$
q \leq \widehat{q} \leq \widehat{q}_{0}:=n\left(\sum_{i=1}^{q}\left(\begin{array}{c}
q \\
i
\end{array}\right)\right), \quad q^{\prime} \leq \hat{q}^{\prime} \leq \widehat{q}_{0}^{\prime}:=\widehat{q}_{0}\left(q^{\prime}+1\right)
$$

so that the components $z_{i}$ and $z_{j}$ correspond to $e^{-h_{i} s}$ and $e^{-h_{j}^{\prime} s}\left(i \in \bar{q}, j \in \bar{q}^{\prime}\right)$, respectively, in a multiargument description, where $s$ and all the components $z_{i}$ and $z_{j}$ are taken as independent variables. The components $\widehat{z}_{i}$ of the extended $\widehat{z}$ are associated with combined delays $\widehat{h}_{i}$ which are formed with all the combinations of sums of the internal delays $h_{i}$ and their respective integer multiplicities. The components $\widehat{z}_{j}$ and associated delays $\widehat{h}_{i}^{\prime}$ are formed with all the above combinations of sums of the internal delays $h_{i}$ and their respective integer multiplicities with the various external delays. The appearance of these delays arises in a natural way in the transfer function and then, roughly speaking, in the input/output data model via direct calculation in the numerator and denominator of (2.4). Intuitively, that means there are usually much more delays in the external system representation than in the internal one due to the evaluation of the adjoint matrix and the determinant and the products in the numerator of (2.4). This feature leads to inequalities with upper bounds in (2.6) so that if identical internal/external delays appear as a result of calculations in the transfer functions (see (2.4)), the resulting coefficients are regrouped so that each of the identical delays appears only once. As intuitive related example, one single internal delay $h$ in the state-space representation generates, up till $n$ commensurate delays, $h_{j}=j h(j \in \bar{n})$ in a single-input single-output transfer function. Then, $\mathbb{R}^{p \times m}(s, z)$, the space of realizable rational transfer $p \times m$ matrices of real coefficients in the complex $\left(\widehat{q}+\widehat{q}^{\prime}+1\right)$ tuple $(s, \widehat{z})$ of numerator and denominator being, respectively, a quasipolynomial matrix and a quasipolynomial, is isomorphic (in the sequel denoted by the symbol " $\approx$ ") to $\mathbb{R}^{p \times m}(s)$ so that there is a natural bijective mapping between each entry $G_{i j}(s)$ and

$$
G_{i j}(s, \hat{z})=\frac{N_{i j}(s, \widehat{z})}{M(s, \hat{z})}=\frac{\sum_{k=0}^{n_{i j}} N_{i j k}(\widehat{z}) s^{k}}{\sum_{k=0}^{n} M_{k}(\widehat{z}) s^{k}}=\frac{\sum_{\ell=0}^{\widehat{q}+\hat{q}^{\prime}} \bar{N}_{i j \ell}(s) \widehat{z}_{\ell}}{\sum_{\ell=0}^{\widehat{q}} \bar{M}_{\ell}(s) \widehat{z}_{\ell}}=\frac{\sum_{k=0}^{n_{i j}} \sum_{\ell=0}^{\hat{q}+\hat{q}^{\prime}} N_{i j k \ell} s^{k} \widehat{z}_{\ell}}{\sum_{k=0}^{n} \sum_{\ell=0}^{\widehat{q}} M_{k \ell} s^{k} \widehat{z}_{\ell}}
$$

whose numerator $N_{i j}(s, \widehat{z})$ and denominator $M(s, \widehat{z})$ are, respectively, polynomials in several variables of respective real coefficients $N_{i j k \ell}$ and $M_{k \ell}$, and $N_{i j k}(\widehat{Z}), M_{k}(\widehat{z}), \bar{N}_{i j k}(s)$, and $\bar{M}_{k}(s)$ are also polynomials in their respective single or multiple arguments.

\section{Analysis of minimal realizations and formal series descriptions}

Note that the numerator and denominator of $G_{i j}(s, \widehat{z})$ are, respectively, in the polynomial additive groups (rings if $p=m) \mathbb{R}^{p \times m}[s, \hat{z}]$ and $\mathbb{R}[s, \widehat{z}]$ generated by $(s, \widehat{z})$. By using a formal Laurent series expansion at $\infty$ in the variable $s$ of the form $G(s, \widehat{z})=\sum_{i=0}^{\infty} H_{i}(\widehat{z}) s^{-i}$ with 
$H_{i}(\widehat{z}) \in \mathbb{R}^{p \times m}[\widehat{z}]$, it follows that $\mathbb{R}^{p \times m}(s, \widehat{z}) \approx \mathbb{R}^{p \times m}[[s]][\widehat{z}]$, the additive group of formal Laurent power series with matrices over $\mathbb{R}^{p \times m}$ at $\infty$ in the polynomial multiple indeterminate defined by the components of the $\widehat{z}$-tuple. Note that the formal series additive group $\mathbb{R}^{p \times m}[[s]][\hat{z}]$ is the completion of the polynomial matrix additive group $\mathbb{R}^{p \times m}[s][\hat{z}]\left(\approx \mathbb{R}^{p \times m}[s, \widehat{z}]\right)$ with respect to the $I$-adic topology, where $I$ is the ideal of the polynomial matrix additive group $\mathbb{R}^{p \times m}[s][\hat{z}]$ generated by the indeterminate complex $\left(\widehat{q}+\hat{q}^{\prime}+1\right)$-tuple $(s, \widehat{z})$. The above discussion is formalized as follows.

Theorem 3.1. The following properties hold for any positive integers $p, m$, and $n$.

(i) $\mathbb{R}^{n \times n}\left[e^{-h_{i} s}: i \in \bar{q} \cup\{0\}\right] \approx \mathbb{R}^{n \times n}\left[z^{I}\right]$ if $h_{i} \in \mathbb{R}_{+} \backslash\{0\}$ for all $i, j \in \bar{q}$, and $h_{i} \neq h_{j}$ for all $i, j(\neq i) \in$ $\bar{q} ; \mathbb{R}^{n \times m}\left[e^{-h_{i}^{\prime} s}: i \in \bar{q}^{\prime} \cup\{0\}\right] \approx \mathbb{R}^{n \times m}\left[z^{E}\right]$.

(ii) $\mathbb{R}^{p \times m}(s, \widehat{z}) \approx \mathbb{R}^{p \times m}[[s]][\hat{z}] \approx \mathbb{R}^{p \times m}[s, \widehat{z}]$ if $h_{i}^{\prime} \in \mathbb{R}_{+} \backslash\{0\}$ for all $i, j \in \bar{q}^{\prime}$, and $h_{i} \neq h_{j}$ for all $i, j(\neq i) \in \bar{q}^{\prime}$.

(iii) $\mathbb{R}^{p \times m}[s][\hat{z}]$ is a dense subspace of $\mathbb{R}^{p \times m}[[s]][\hat{z}]$, which is a complete topological additive group with respect to the $\mathbf{I}$-adic topology, where $\mathbf{I}$ is the ideal of the additive group $\mathbb{R}^{p \times m}[s][\hat{z}]$ generated by the indeterminate complex $\left(\widehat{q}+\widehat{q}^{\prime}+1\right)$-tuple $(s, \widehat{z})$.

Note that the isomorphisms of Theorem 3.1(i) are useful to formulate controllability/observability and minimal realizations for the dynamic system (2.1)-(2.2) since the only delays which are zero are $h_{0}=h_{0}^{\prime}=0$ and all the remaining internal delays are pairwise distinct while all the remaining external delays are pairwise distinct as well.

From Theorem 3.1(i), the following bijections may be established:

$$
\begin{aligned}
& \sum_{i=0}^{q} A_{i} e^{-h_{i} s} \longleftrightarrow A\left(z^{I}\right):=\sum_{i=0}^{q} A_{i} z_{i}^{I} \in \mathbb{R}^{n \times n}\left[z^{I}\right] \\
& \sum_{i=0}^{q^{\prime}} B_{i} e^{-h_{i}^{\prime} s} \longleftrightarrow A\left(z^{E}\right):=\sum_{i=0}^{q} A_{i} z_{i}^{E} \in \mathbb{R}^{n \times n}\left[z^{I}\right]
\end{aligned}
$$

so that via (3.1) the controllability and observability matrices of the $n$th realization (2.1)-(2.2) result:

$$
\begin{gathered}
C_{n}(A(z), B(z))=C_{n}\left(A\left(z^{I}\right), B\left(z^{E}\right)\right):=\left(B\left(z^{E}\right), A\left(z^{I}\right) B\left(z^{E}\right), \ldots, A^{n-1}\left(z^{I}\right) B\left(z^{E}\right)\right), \\
O_{n}(C, A(z))=O_{n}\left(C, A\left(z^{I}\right)\right):=\left(C^{T}, A^{T}\left(z^{I}\right) C^{T}, \ldots, A^{n-1 T}\left(z^{I}\right) C^{T}\right)^{T}
\end{gathered}
$$

in $\mathbb{R}^{n \times(n+m)}[\hat{z}]$ and $\mathbb{R}^{p \times(p+m)}[\hat{z}]$, respectively. Note that if some of the above matrices are full rank, then the state-space realization (2.1)-(2.2) is controllable (resp., observable) in an additive group. However, the respective full-rank conditions are not necessary for controllability / observability since the additive group isomorphism defined by (3.1) does not preserve the metric and topologic properties. In particular, the loss of rank of any of the polynomial matrices (see (3.2)) for some delays $h_{i} \geq 0(i \in \bar{q}), h_{j} \geq 0\left(j \in \bar{q}^{\prime}\right)$, and $h_{0}=h_{0}^{\prime}=0$ in the indeterminate $z$ does not imply that the rank is lost for some complex indeterminate $s$ satisfying the constraints $z_{i}=e^{-h_{i} s}, z_{q+j}=e^{-h_{j}^{\prime} s}\left(i \in \bar{q}, j \in \bar{q}^{\prime}\right)$ for some predefined delays.Define the following controllability and observability testing sets $S_{C_{n}}(\mathbf{h})$ and $S_{O_{n}}(\mathbf{h})$, respectively, depending on the real 
$\left(q+q^{\prime}\right)$-tuple of delays:

$$
\mathbf{h}=\left(h_{1}, h_{2}, \ldots, h_{q}, h_{q+1}=h_{1}^{\prime}, h_{q+2}=h_{2}^{\prime}, \ldots, h_{q+q^{\prime}}=h_{q^{\prime}}^{\prime}\right) \in \mathbb{R}_{+}^{q+q^{\prime}},
$$

which is the closed first orthant in $\mathbb{R}^{q+q^{\prime}}$, and the associated sets of delays are $H_{u c}$ and $H_{u o}$, where controllability and, respectively, observability are lost:

$$
\begin{aligned}
& S_{C_{n}}(\mathbf{h}):=\left\{z=\left(z_{1}, z_{2}, \ldots, z_{q}\right) \in \mathbf{C}^{q+q^{\prime}}: z_{i}=\left|z_{i}\right|_{<\theta_{i}}=e^{-h_{i} s}, \sigma_{C}=\frac{\ln \left|z_{i}\right|}{h_{i}} \in \mathbb{R},\right. \\
&\left.\omega_{C}=\frac{\operatorname{tg}\left(\theta_{i}\right)}{h_{i}} \in \mathbb{R} \forall i \in \overline{q+q^{\prime}}, \operatorname{rank}\left[C_{n}\left(A\left(z^{I}\right), B\left(z^{E}\right)\right)\right]<n\right\}, \\
& S_{O_{n}}(\mathbf{h}):=\left\{z=\left(z_{1}, z_{2}, \ldots, z_{q}\right) \in \mathbf{C}^{q}: z_{i}=\left|z_{i}\right|_{<\theta_{i}}=e^{-h_{i} s}, \sigma_{O}=\frac{\ln \left|z_{i}\right|}{h_{i}} \in \mathbb{R},\right. \\
&\left.\omega_{O}=\frac{\operatorname{tg}\left(\theta_{i}\right)}{h_{i}} \in \mathbb{R} \forall i \in \bar{q}, \operatorname{rank}\left[O_{n}\left(C, A\left(z^{I}\right)\right)\right]<n\right\}, \\
& H_{u c}:=\left\{\mathbf{h} \in \mathbb{R}_{+}^{q+q^{\prime}}: S_{C_{n}}(\mathbf{h}) \neq \varnothing\right\}, \\
& H_{u o}:=\left\{\mathbf{h} \in \mathbb{R}_{+}^{q+q^{\prime}}: S_{O_{n}}(\mathbf{h}) \neq \varnothing\right\} .
\end{aligned}
$$

Note that if the full ranks in the polynomial matrices (see (3.2)) are lost for some $\mathbf{h}$ such that the respective testing set in (3.4) is nonempty, then controllability (resp., observability) in an additive group becomes lost for the corresponding set of delays. If the full-rank property in the polynomial matrices (see (3.2)) is lost for some $\mathbf{h}$ such that the respective testing set in (3.4) is empty, then controllability (resp., observability) in an additive group holds for the corresponding set of delays. If the sets $S_{C_{n}}(\mathbf{h})$ and $S_{O_{n}}(\mathbf{h})$, respectively, are empty for any $\mathbf{h} \in$ $\mathbb{R}_{+}^{q+q^{\prime}}$, then the system is controllable (resp., observable) in an additive group independent of the delays. Note directly that

$$
\mathbf{h} \in H_{u c} \Longleftrightarrow S_{C_{n}}(\mathbf{h}) \neq \varnothing ; S_{C_{n}}(\mathbf{h})=\varnothing \quad \forall \mathbf{h} \in \mathbb{R}_{+}^{q+q^{\prime}} \Longleftrightarrow H_{u c}=\varnothing,
$$

and similar assertions are applicable to the sets $S_{O_{n}}(\mathbf{h})$ and $H_{u o}$. Then, the following definitions on spectral controllability and observability are provided. Then, a related result is given as a formal statement of the above informal discussions, which states formally the equivalences between spectral controllability (observability) and controllability (observability).

Definition 3.2. The dynamic system (2.1)-(2.2) is spectrally controllable if there exists a statefeedback control law $u: \mathbb{R}_{+} \times \mathbb{R}^{n} \rightarrow \mathbb{R}^{m}$, fulfilling $u(t)=0$ for all $t \in \mathbb{R}_{-}:=\mathbb{R} \backslash \mathbb{R}_{+}$, such that $U(s)=\operatorname{Lap}(u(t))$ exists for any given prefixed suited characteristic closed-loop polynomial:

$$
\sum_{i=0}^{n} \sum_{j=0}^{q+q^{\prime}} f_{i j} s^{i} e^{-h_{j} s}=\operatorname{Det}\left(s I_{n}-\sum_{i=0}^{q} A_{i} e^{-h_{i} s}-\sum_{i=0}^{q^{\prime}} B_{i} e^{-h_{i}^{\prime} s} U(s)\right) .
$$

If the above property holds for any given vector of delays $\mathbf{h}$, then the system is spectrally controllable independent of the delays. 
Definition 3.3. The dynamic system (2.1)-(2.2) is spectrally observable if its dual is spectrally controllable, that is, if there exists a state-feedback control law $u: \mathbb{R}_{+} \times \mathbb{R}^{n} \rightarrow \mathbb{R}^{p}$, fulfilling $u(t)=0$ for all $t \in \mathbb{R}_{-}:=\mathbb{R} \backslash \mathbb{R}_{+}$, such that $U(s)=\operatorname{Lap}(u(t))$ exists for any given prefixed suited characteristic closed-loop polynomial:

$$
\sum_{i=0}^{n} \sum_{j=0}^{q^{\prime}} f_{i j} s^{i} e^{-h_{j} s}=\operatorname{Det}\left(s I_{n}-\sum_{j=0}^{q} A_{i}^{T} e^{-h_{i} s}-C^{T} U(s)\right)
$$

If the above property holds for any given vector of internal delays, then the system is spectrally observable independent of the delays.

Controllability and observability in rings are defined in parallel to their above spectral versions in the complex indeterminates $z_{i}$ (which replace $e^{-h_{i} s}$ in Definitions 3.2 and 3.3) which are considered to be mutually independent; this is not true since they are related by the Laplace transform indeterminate $s$. Therefore, controllability and observability in rings are sufficient (but not necessary) for their corresponding spectral versions to hold in the context of time-delay systems. Note that observability is defined by duality in order to simplify the description. On the other hand, note that the spectral definitions of controllability and observability are established in terms of the ability of arbitrary coefficient assignment of the characteristic closed-loop polynomial (or that of the dual system) through some realizable control law. These definitions are equivalent to the classical spectral definitions for time-delay timeinvariant systems which were stated in equivalent terms via Popov-Belevitch-Hautus controllability/observability tests (see, e.g., [35-38]). Such tests are used in Theorem 3.4 in terms of being necessary and sufficient to guarantee both properties. Finally, note that the real controllability/observability (in terms of necessary and sufficient conditions for prefixed assignment of closed-loop modes) has to be stated in the spectral context. Alternative classical formulations in rings provide only sufficient conditions for controllability / observability of the dynamic system since characteristic quasipolynomial $s$ is treated as if it were polynomial of several independent variables, that is, as if exponential terms of the form $z=e^{-h s}$ were independent of $s$. In other words, conditions implying loss of controllability/observability appear by considering the arguments $s$ and $z$ as independent variables. Such conditions are spurious and have to be removed in the cases where $z \neq e^{-h s}$.

Theorem 3.4. The following properties hold.

(i) The state-space realization (2.1)-(2.2) is spectrally controllable for some given $\mathbf{h} \in \mathbb{R}_{+}^{q+q^{\prime}}$ in the first orthant if and only if $\operatorname{rank}\left[s I_{n}-\sum_{i=0}^{q} A_{i} e^{-h_{i} s}, \sum_{i=0}^{q^{\prime}} B_{i} e^{-h_{i}^{\prime} s}\right]=n$ for all $s \in \mathrm{C}$. The state-space realization system (2.1)-(2.2) is spectrally observable if and only if $\operatorname{rank}\left[s I_{n}-\sum_{i=0}^{q} A_{i}^{T} e^{-h_{i}^{\prime} s}, C^{T}\right]=$ $n$ for all $s \in \mathbf{C}$. The state-space realization (2.1)-(2.2) is minimal of order $n$ if and only if it is spectrally controllable and spectrally observable [27], that is,

$$
\operatorname{rank}\left[s I_{n}-\sum_{i=0}^{q} A_{i} e^{-h_{i} s}, \sum_{i=0}^{q^{\prime}} B_{i} e^{-h_{i}^{\prime} s}\right]=\left[s I_{n}-\sum_{i=0}^{q} A_{i}^{T} e^{-h_{i}^{\prime} s}, C^{T}\right]=n \quad \forall s \in \mathbf{C} .
$$

Both full-rank conditions hold simultaneously; then the state-space realization (2.1)-(2.2) is minimal and the converse is also true. 
(ii) The state-space realization (2.1)-(2.2) is controllable in a ring independent of the delays, that is, for any $\mathbf{h} \in \mathbb{R}_{+}^{q+q^{\prime}}$, if $\operatorname{rank}\left[C_{n}(A(z), B(z))\right]=n$ for all $z \in C^{q+q^{\prime}}$, while the converse is not true in general. The state-space realization (2.1)-(2.2) is observable in a ring independent of the delays if $\operatorname{rank}\left[O_{n}(C, A(z))\right]=n$ for all $z \in C^{q+q^{\prime}}$, and the converse is not true. The state-space realization (2.1)-(2.2) is minimal of order $n$ independent of the delays if

$$
\operatorname{rank}\left[C_{n}(A(z), B(z))\right]=\operatorname{rank}\left[O_{n}(C, A(z))\right]=n \quad \forall z \in C^{q+q^{\prime}},
$$

while the converse is not true in general.

(iii) The state-space realization (2.1)-(2.2) is controllable (resp., observable) in a ring independent of the delays if and only if $S_{C_{n}}(\mathbf{h})=\varnothing$ for any $\mathbf{h} \in \mathbb{R}_{+}^{q+q^{\prime}}\left(\right.$ resp., $S_{O_{n}}(\mathbf{h})=\varnothing$ for any $\mathbf{h} \in \mathbb{R}_{+}^{q+q^{\prime}}$ ). The state-space realization (2.1)-(2.2) is minimal if and only if $S_{C_{n}}(\mathbf{h})=S_{O_{n}}(\mathbf{h})=\varnothing$ for any $\mathbf{h} \in \mathbb{R}_{+}^{q+q^{\prime}}$, that is, if and only if it is both controllable and observable in a ring independent of the delays.

(iv) The state-space realization (2.1)-(2.2) is controllable (resp., observable) in a ring for any given $\mathbf{h} \in \mathbb{R}_{+}^{q+q^{\prime}}$ if and only if $S_{C_{n}}(\mathbf{h})=\varnothing\left(\right.$ resp., $\left.S_{O_{n}}(\mathbf{h})=\varnothing\right)$. The state-space realization $(2.1)-(2.2)$ is minimal if and only if $S_{C_{n}}(\mathbf{h})=S_{O_{n}}(\mathbf{h})=\varnothing$, that is, if and only if it is both controllable and observable in a ring.

(v) The state-space realization (2.1)-(2.2) is controllable (resp., observable) in a ring being either dependent on $\mathbf{h} \in \mathbb{R}_{+}^{q+q^{\prime}}$ or independent of the delays if and only if it is spectrally controllable (resp., spectrally observable) being either dependent on or independent of the delays.

(vi) The state-space realization (2.1)-(2.2) is controllable (resp., observable) in a ring independent of the delays, and equivalently spectrally controllable (resp., spectrally observable) independent of the delays if and only if $H_{u c}=\varnothing\left(\right.$ resp., $\left.H_{u o}=\varnothing\right)$ with $H_{u c}$ and $H_{u o}$ defined in (3.4). The state-space realization (2.1)-(2.2) is minimal independent of the delays of order $n$ if and only if $H_{u c} \cup H_{u o}=\varnothing$, that is, if and only if it is both controllable and observable in a ring independent of the delays.

The state-space realization (2.1)-(2.2) is spectrally uncontrollable (resp., spectrally unobservable) for a given $\mathbf{h} \in \mathbb{R}_{+}^{q+q^{\prime}}$ if and only if $\mathbf{h} \in H_{u c}$ (resp., $\mathbf{h} \in H_{u o}$ ) and equivalently if and only if $S_{C_{n}}(\mathbf{h}) \neq \varnothing$ (resp., $\left.S_{O_{n}}(\mathbf{h}) \neq \varnothing\right)$.

Proof. (i) It is a direct generalization of the Popov-Belevitch-Hautus rank controllability / observability tests [39] to the case of point time delays. The result for controllability follows directly by taking Laplace transforms in (2.1) with initial condition $\varphi \equiv 0$. The parallel result for observability follows directly by taking Laplace transforms in (2.1)-(2.2) with $u \equiv 0$ and nonzero point initial conditions at $t=0$, namely, $x_{0}=x(0)=\varphi(0) \neq 0$.

(ii) Note that for any complex matrices $A$ and $B$ and compatible orders, $\operatorname{rank}\left[s I_{n}-A, B\right]=$ $n$ for all $s \in \mathrm{C}$ if and only if $\operatorname{rank}\left[C_{n}(A, B)\right]=n$ from Popov-Belevitch-Hautus rank controllability test for the linear time-invariant delay-free case. Thus, for polynomial matrices $A(z)$ and $B(z)$ in several complex variables, $\operatorname{rank}\left[s I_{n}-A(z), B(z)\right]=n$ for all $s \in \mathbf{C}$ for some given complex $\left(q+q^{\prime}\right)$-tuple $z$ if and only if $\operatorname{rank}\left[C_{n}(A(z), B(z))\right]=n$ for some given complex $\left(q+q^{\prime}\right)$-tuple $z$, since for each $z, A(z)$ and $B(z)$ are complex matrices.

If $\operatorname{rank}\left[C_{n}(A(z), B(z))\right]=n$ for any complex $\left(q+q^{\prime}\right)$-tuple $z$, then the property (ii) follows from the ring isomorphisms of Theorem 3.1, made explicit in (3.1). A similar proof follows for observability. Since a loss of full rank at some $z$ does not necessarily imply that all of its components satisfy $z_{i}=e^{-h_{i} s}$ for all $i \in \overline{q+q^{\prime}}$ and $h_{q+i}=h_{i}^{\prime}$ for all $i \in \overline{q^{\prime}}$, then the controllability / observability conditions are not necessary.

(iii)-(iv) If $S_{C_{n}}(\mathbf{h})$, defined in (3.4), is empty, then $\operatorname{rank}\left[C_{n}(A(z), B(z))\right]=n$ for all complex $\left(q+q^{\prime}+1\right)$-tuple $(s, z)$ such that the constraints $z_{i}=e^{-h_{i} s}$ for all $i \in \overline{q+q^{\prime}}$ and $h_{q+i}=h_{i}^{\prime}$ 
for all $i \in \bar{q}^{\prime}$ are satisfied. This proves necessity. Sufficiency follows directly from (ii). A similar reasoning applies to observability with $S_{C_{n}}(\mathbf{h})$ being empty and $\operatorname{rank}\left[O_{n}(C, A(z))\right]=n$.

(v) The results (i)-(v) imply for any given $\mathbf{h} \in \mathbb{R}_{+}^{q+q^{\prime}}$ that

$$
\begin{aligned}
& \operatorname{rank}\left[s I_{n}-\sum_{i=0}^{q} A_{i} e^{-h_{i} s}, \sum_{i=0}^{q^{\prime}} B_{i} e^{-h_{i}^{\prime} s}\right]=n \quad \forall s \in \mathbf{C} \\
& \Longleftrightarrow \operatorname{rank}\left[C_{n}(A(z), B(z))\right]=n \quad \forall z \notin S_{C_{n}}(\mathbf{h}) \Longleftrightarrow S_{C_{n}}(\mathbf{h})=\varnothing, \\
& \operatorname{rank}\left[s I_{n}-\sum_{i=0}^{q} A_{i}^{T} e^{-h_{i} s}, C^{T}\right]=n \quad \forall s \in \mathbf{C} \\
& \Longleftrightarrow \operatorname{rank}\left[O_{n}(C, A(z))\right]=n \quad \forall z \notin S_{O_{n}}(\mathbf{h}) \Longleftrightarrow S_{O_{n}}(\mathbf{h})=\varnothing .
\end{aligned}
$$

(vi) It follows directly from properties (iii)-(v), since the sets $H_{u c}$ and $H_{u o}$ are empty, that there is no vector of delays such that the respective spectral controllability and observability tests fail resulting in the corresponding matrices being rank-defective. As a result, the system is controllable (resp., observable) independent of the delays.

Remark 3.5. Note that from the definition of $S_{C_{n}}(\mathbf{h})=\varnothing$, it is only necessary to consider $z-\left(q+q^{\prime}\right)$ tuples with all of their components satisfying simultaneously either $\left|z_{i}\right| \geq 1$ or $\left|z_{i}\right|<1$ for all $i \in$ $\overline{q+q^{\prime}}$, which satisfy furthermore $g(s, z):=\operatorname{Det}\left(s I_{n}-A\left(z^{I}\right)\right)=0\left(\right.$ since $\operatorname{rank}\left[C_{n}(A(z), B(z))\right]=n$ for all $z$ such that $g(s, z) \neq 0$ ) in order to test $S_{C_{n}}(\mathbf{h})=\varnothing$. Similar considerations apply for testing $S_{O_{n}}(\mathbf{h})=\varnothing$. This facilitates the way of performing the controllability/observability tests in practice.

Now, consider the sequence $H^{\tau}(\widehat{z}):=\left\{H_{i}(\widehat{z})\right\}_{1}^{\tau}$ with $\tau \in \mathbb{N}$ which defines the $\tau$-finite block complex Hankel matrix

$$
H(i, \tau+1-i, \widehat{z}):=\left[\begin{array}{ccc}
H_{1}(\widehat{z}) & \cdots & H_{\tau+1-i}(\widehat{z}) \\
\vdots & \vdots & \vdots \\
H_{i}(\widehat{z}) & \cdots & H_{\tau}(\widehat{z})
\end{array}\right\rfloor=\left[\begin{array}{ccc}
C B\left(z^{E}\right) & \cdots & C A^{\tau-i}\left(z^{I}\right) B\left(z^{E}\right) \\
\vdots & \vdots & \vdots \\
C A^{i-1}\left(z^{I}\right) B\left(z^{E}\right) & \cdots & C A^{\tau-1}\left(z^{I}\right) B\left(z^{E}\right)
\end{array}\right\rfloor .
$$

For $\tau=\infty$, the infinite Hankel block matrix is $H_{G}(\widehat{z}):=\operatorname{Block} \operatorname{Matrix}\left(H_{i+j-1}(\widehat{z})\right)_{i, j \in \mathbb{N}}$. From (3.2) and (3.11), the subsequent technical result holds directly, where the generic rank (denoted as gen rank) of the (.)-polynomial matrix $(\cdot)$ is its maximum rank reached on the overall set of values of its argument. Note that there is a natural surjective mapping $\mathbf{C}^{q+q^{\prime}} \rightarrow \mathbf{C}^{\hat{q}+\hat{q}^{\prime}}$ which maps each argument $z$ into one corresponding $\widehat{z}(z)$; it is irrelevant to replace the argument $\widehat{z}$ by its preimage $z$ in all of the subsequent notations and related discussions about controllability/observability in the appropriate rings of polynomials, quasipolynomials, or series. Therefore, both arguments $z$ and $\hat{z}$ are used indistinctly where appropriate according to convenience for clarity. 
Lemma 3.6. The following properties hold independent of the delays.

(i) $H(i, \tau+1-i, \widehat{z})=O_{i}\left(C, A\left(z^{I}\right)\right) C_{\tau+1-i}\left(A\left(z^{I}\right), B\left(z^{E}\right)\right) \forall \widehat{z} \in \mathbf{C}^{\hat{q}+\widehat{q}^{\prime}}$.

(ii) $\operatorname{rank}[H(i, \tau+1-i, \widehat{z})] \leq \operatorname{Min}(i, \tau+1-i, n) \forall \hat{z} \in \mathbf{C}^{\hat{q}+\hat{q}^{\prime}}$.

(iii) $\operatorname{rank}[H(i, \tau+1-i, \widehat{z})] \leq n$ for any $\tau, i \in \mathbb{N}$ with $\tau \geq n+i-1, i \geq n$ for all $\hat{z} \in \mathbf{C}^{\hat{q}+\hat{q}^{\prime}}$, where $n$ is the order of the state-space realization (2.1)-(2.2).

(iv) $\operatorname{rank}\left[H_{G}(\widehat{z})\right] \leq \operatorname{gen} \operatorname{rank}_{\tau \geq n+i-1, i \geq n, \hat{z} \in \mathbf{C}^{\hat{q}+\hat{q}^{\prime}}}\left[H_{G}(\widehat{z})\right]=\operatorname{gen}^{\operatorname{rank}_{\tau \geq n+i-1, i \geq n,} \bar{z} \in \mathbf{C}^{\hat{q}+\hat{q}^{\prime}}}[H(i, \tau+$ $1-i, \widehat{z})] \leq n \forall \widehat{z} \in \mathbf{C}^{\hat{q}+\widehat{q}^{\prime}}$.

Lemma 3.6 establishes that the rank of a $\tau$-finite or infinite block Hankel matrix is always finite and it cannot exceed the order of given state-space realization.

Theorem 3.7. Consider two state-space realizations of the transfer matrix of (2.1)-(2.2):

$$
\begin{aligned}
& R:=\left(A_{0}, A_{i}(i \in \bar{q}), B_{0}, B_{j}\left(j \in \bar{q}^{\prime}\right), C, D\right), \\
& \bar{R}:=\left(\bar{A}_{0}, \bar{A}_{i}(i \in \bar{q}), \bar{B}_{0}, \bar{B}_{j}\left(j \in \bar{q}^{\prime}\right), \bar{C}, \bar{D} \equiv D\right)
\end{aligned}
$$

of respective orders $n$ (minimal) and $\bar{n}>n$. Then, the following properties hold independent of the delays.

(i)

$$
\begin{gathered}
n \leq \underset{\tau \geq \bar{n}+i-1, i \geq \bar{n}, \hat{z} \in \mathbf{C}^{+}+\hat{q}^{\prime}}{\operatorname{gen} \operatorname{rank}}\left[\bar{H}_{G}(\widehat{z})\right] \leq \underset{\tau \geq \bar{n}+i-1, i \geq \bar{n}, \hat{z} \in \mathbf{C}^{q}+\bar{q}^{\prime}}{\operatorname{gen} \operatorname{rank}}[\bar{H}(i, \tau+1-i, \widehat{z})] \leq \bar{n}, \\
\operatorname{Min}\left(\underset{\tau \geq n+i-1, i \geq n, \hat{z} \in S_{C_{\bar{n}}}(\mathbf{h})}{\operatorname{gen} \operatorname{rank}}[\bar{H}(i, \tau+1-i, \widehat{z})], \underset{\tau \geq n+i-1, i \geq n, \hat{z} \in S_{\bar{O}_{\bar{n}}}(\mathbf{h})}{\operatorname{gen~rank}}[\bar{H}(i, \tau+1-i, \widehat{z})]\right)<\bar{n}, \\
n \leq \operatorname{Min}\left(\underset{\tau \geq n, \hat{z} \in S_{C_{\bar{n}}}(\mathbf{h})}{\operatorname{gen} \operatorname{rank}}\left[C_{\tau}\left(\bar{A}\left(z^{I}\right), \bar{B}\left(z^{E}\right)\right)\right], \underset{\tau \geq n, \hat{z} \in S_{O_{\bar{n}}}(\mathbf{h})}{\operatorname{gen} \operatorname{rank}}\left[O_{\tau}\left(\bar{A}\left(z^{I}\right), \bar{B}\left(z^{E}\right)\right)\right]\right)<\bar{n} \quad \forall \mathbf{h} \in \mathbb{R}_{+}^{q+q^{\prime}} .
\end{gathered}
$$

(ii)

$$
\begin{aligned}
\underset{\tau \geq n+i-1, i \geq n \in N, \hat{z} \in C^{\hat{q}+\hat{q}^{\prime}}}{\text { gen rank }}[H(i, \tau+1-i, \widehat{z})] & =\underset{\tau \geq n, \hat{z} \in \mathbf{C}^{\hat{q}+\hat{q}^{\prime}}}{\operatorname{gen} \operatorname{rank}}\left[C_{\tau}\left(A\left(z^{I}\right), B\left(z^{E}\right)\right)\right] \\
& =\underset{\tau \geq n, \hat{z} \in \mathbf{C}^{\hat{q}+\hat{q}^{\prime}}}{\operatorname{gen} \operatorname{rank}}\left[O_{i}\left(C, A\left(z^{I}\right)\right)\right]=n .
\end{aligned}
$$

(iii) None of the following conditions can hold for a complex function $z: \mathbf{C} \times \mathbb{R}_{+}^{q+q} \rightarrow \mathbf{C}^{q+q^{\prime}}$ defined by

$$
z(s, \mathbf{h})=\left(z^{I}(s, \mathbf{h}), z^{E}(s, \mathbf{h})\right)=\left(e^{-h_{1} s}, \ldots, e^{-h_{q} s}, e^{-h_{1}^{\prime} s}, \ldots, e^{-h_{q}^{\prime} s}\right)
$$

associated with internal and external delays $h_{i}(i \in \bar{q}), h_{q+j}=h_{j}^{\prime}\left(j \in \bar{q}^{\prime}\right)$. Then,

$$
\begin{array}{rlrl}
\operatorname{rank}\left[H_{G}(\widehat{z})\right] & <n, & \\
\operatorname{rank}[H(i, \tau+1-i, \widehat{z})] & <n & \text { for any } \tau(\geq n+i-1), i(\geq n) \in \mathbb{N}, \\
\operatorname{rank}\left[C_{\tau}\left(A\left(z^{I}\right), B\left(z^{E}\right)\right)\right]<n & \forall \tau(\geq n) \in \mathbb{N}, \\
\operatorname{rank}\left[O_{\tau}\left(C, A\left(z^{I}\right)\right)\right]<n & \forall \tau(\geq n) \in \mathbb{N} .
\end{array}
$$


Proof. Properties (i)-(ii) follow directly from the factorization of Lemma 3.6(i) and the rank constraints in Lemmas 3.6(ii) and 3.6(iii) since the ranks of the controllability and observability matrices never exceed the order of a minimal realization, and on the other hand, the generic ranks of the observability and controllability matrices equalize the order of any minimal realization. This proves that the generic rank is upper-bounded by $n$. The fact that it is identical to a minimum $n$ follows from the contradiction which would arise if

$$
\underset{\tau \geq n, \in \mathbf{C}^{++q^{\prime}}}{\operatorname{gen} \operatorname{rank}}\left[C_{\tau}\left(A\left(z^{I}\right), B\left(z^{E}\right)\right)\right]>\underset{\tau \geq n, z \in S_{C_{n}}(\mathbf{h}) \cup S_{O_{n}}(\mathbf{h})}{\operatorname{rank}}\left[C_{\tau}\left(A\left(z^{I}\right), B\left(z^{E}\right)\right)\right] ;
$$

then there would exist $\underline{n}(<n) \in \mathbb{N}$ such that

$$
\underset{\tau \geq \underline{n}, z \in \mathbf{C}^{q+q^{\prime}}}{\operatorname{gen} \operatorname{rank}}\left[C_{\tau}\left(A\left(z^{I}\right), B\left(z^{E}\right)\right)\right]=\underset{\tau \geq \underline{n}, z \in S_{C_{\underline{\underline{n}}}}(\mathbf{h}) \cup S_{O_{\underline{\underline{n}}}}(\mathbf{h})}{\operatorname{rank}}\left[C_{\tau}\left(A\left(z^{I}\right), B\left(z^{E}\right)\right)\right]=\underline{n},
$$

and then the order of the square polynomial matrix $A\left(Z^{I}\right)$ and, as a result, that of matrices $A_{0}, A_{i}(i \in \bar{q})$ would be $\underline{n}<n$. Since $n$ is the order of a minimal realization, $S_{C_{n}}(\mathbf{h}) \cup$ $S_{O_{n}}(\mathbf{h})=\varnothing$ from Theorem 3.4(iv), which implies and is implied by $\operatorname{rank}\left[C_{\tau}\left(A\left(z^{I}\right), B\left(z^{E}\right)\right)\right]=$ $\operatorname{rank}\left[O_{\tau}\left(C, A\left(z^{I}\right)\right)\right]=n$ for all $\tau(\geq n) \in \mathbb{N}$ and all $z: \mathbf{C} \times \mathbb{R}_{+}^{q+q} \rightarrow \mathbf{C}^{q+q^{\prime}}$ defined by $z(s, \mathbf{h})=$ $\left(z^{I}(s, \mathbf{h}), z^{E}(s, \mathbf{h})\right)=\left(e^{-h_{1} s}, \ldots, e^{-h_{q} s}, e^{-h_{1}^{\prime} s}, \ldots, e^{-h_{q}^{\prime} s}\right)$. This proves property (iii).

\section{Synthesis of minimal realizations}

The problems of synthesis of a minimal realization or a minimal partial realization are formulated in terms of finding a state-space realization such that it matches a certain transfer matrix which is formally identical to a series Laurent expansion at $\infty$. Thus, given the sequence $H^{\tau}(\widehat{z}):=\left\{H_{i}(\widehat{z})\right\}_{0}^{\tau}$ with $\tau(\leq \infty) \in \mathbb{N}$, find matrices $A_{i} \in \mathbb{R}^{n \times n}(i \in \bar{q} \cup\{0\}), B_{i} \in \mathbb{R}^{n \times m}\left(i \in \bar{q}^{\prime} \cup\{0\}\right)$, and $C \in \mathbb{R}^{p \times n}$ provided that they exist such that the following matching condition holds independent of the delays either for all $\tau \in \mathbb{N}$ (minimal synthesis problem) or for some finite $\tau \in \mathbb{N}$ (minimal partial realization problem):

$$
G(s, \widehat{z}):=C\left(s I-A_{0}-\sum_{i=1}^{q} A_{i} z_{i}^{I}\right)^{-1}\left(B_{0}+\sum_{i=1}^{q^{\prime}} B_{i} z_{i}^{E}\right)+D=\sum_{i=0}^{\infty} H_{i} s^{-i}=\sum_{i=0}^{\tau} H_{i} s^{-i}+0\left(s^{-\tau-1}\right),
$$

where $G(s, \widehat{z}) \in \mathbb{R}^{p \times m}(s, \widehat{z}) \approx \mathbb{R}^{p \times m}[[s]][\hat{z}] \ni \sum_{i=0}^{\tau \leq \infty} H_{i}(\widehat{z}) s^{-i}$ such that $n$ is as small as possible. If the minimal (resp., partial minimal) realization synthesis problem is solvable (i.e., it has a solution), then by making the changes $z_{i}=e^{-h_{i} s}, z_{q+j}=e^{-h_{j}^{\prime} s}\left(i \in \bar{q}, j \in \bar{q}^{\prime}\right)$, a state-space realization (2.1)-(2.2) is obtained so that (4.1) holds for $\tau \in \mathbb{N}$ (resp., for some natural number $\tau<\infty$ ). If the problem is solvable, then there are infinitely many minimal (resp., partial minimal) realizations satisfying it, since any nonsingular state transformation preserves the transfer matrix. In what follows, the result, where the McMillan degree (denoted by $\mu$ ) of a rational transfer matrix coincides with that of the rank of the infinite associated block Hankel matrix for $\widehat{z} \in \mathrm{C}^{q+q^{\prime}}$ (which is also called the McMillan degree of this one), is extended from the delay-free case. The block Hankel matrices are now polynomial matrices. The idea is extended also to truncated finite block Hankel matrices, and it concludes that such a degree equalizes the order of minimal (or partial minimal) state-space realizations. 
Theorem 4.1. The following properties hold.

(i) The McMillan degree $n=\mu(G(s, \mathbf{h}))$ of the transfer matrix $G(s, \mathbf{h})$ is the unique order of any minimal realization of $G(s, \mathbf{h})$ and satisfies the following constraints for any set of delays being components of some given $\mathbf{h} \in \mathbb{R}_{+}^{q+q^{\prime}}$ :

$$
\begin{aligned}
\infty>n(\mathbf{h}) & =\mu(G(s, \mathbf{h})) \\
& =\operatorname{Max}_{\tau \in \mathbb{N}}\left(\underset{z \in S_{C_{\tau}}(\mathbf{h}) \cup S_{O_{\tau}}(\mathbf{h})}{\mu}\left(H^{\tau}(z)\right)\right) \\
& =\operatorname{Max}_{\tau \in \mathbb{N}}\left(\sum_{i+j=\tau+1} \operatorname{rank} H(i, j, z)-\sum_{i+j \tau} \operatorname{rank} H(i, j, z): z \in S_{C_{\tau}}(\mathbf{h}) \cup S_{0_{\tau}}(\mathbf{h})\right) \\
& =\operatorname{Max}_{z \in S_{C_{\infty}}(\mathbf{h}) \cup S_{S_{\infty}}(\mathbf{h})}(\operatorname{rank} H(i, j, z): n \leq i \in \mathbb{N}, i+n-1 \leq \tau \in \mathbb{N}) .
\end{aligned}
$$

(ii) The state-space dimension $n_{\tau}(\mathbf{h})(\tau \in \mathbb{N})$ of any minimal partial realization satisfies

$$
\infty>n_{\tau}(\mathbf{h})=\operatorname{Max}_{\tau \in \mathbb{N}}\left(\underset{z \in S_{C_{\tau}}(\mathbf{h}) \cup S_{O_{\tau}}(\mathbf{h})}{\mu}(H(i, \tau+1-i, z)): i \in \bar{\tau}\right),
$$

where $n_{\tau}(\mathbf{h})=n(\mathbf{h})$, and then the minimal partial realization is a minimal realization for all $\tau\left(\geq \tau_{0}\right) \in$ $\mathbb{N}$ and sufficiently large finite $\tau_{0} \in N$ with

$$
n_{\tau}(\mathbf{h})=n_{\tau_{0}}(\mathbf{h})=n(\mathbf{h})=\underset{z \in S_{\mathcal{C}_{\infty}}(\mathbf{h}) \cup S_{0_{\infty}}(\mathbf{h})}{\operatorname{Min}_{(}}\left(\operatorname{rank} H(i, \tau+1-i, z): \tau_{0} \leq i \in N, i+\tau_{0}-1 \leq \tau \in N\right) .
$$

(iii) Redefine by simplicity the delays according to $h_{q+i}=h_{i}^{\prime}\left(i \in \bar{q}^{\prime}\right)$. Define $\mathbf{h}^{00}=0$ and let $\mathbf{h}^{\mathbf{i} 0}$ be defined with $h_{i} \neq 0$ and $h_{j}=0(j \neq i)$ for $\left(i \in q+q^{\prime}\right)$. Assume that $n\left(\mathbf{h}^{\mathbf{i} 0}\right)=n_{i 0}=n_{0}$ (some constant $n_{0}$ in $\mathbb{N}$ ) for all $i \in \bar{q}$, where

$$
\begin{aligned}
n_{i 0} & :=\operatorname{Min}_{\tau_{i} \in \mathbb{N}}\left(\sum_{i+j=\tau_{i 0}+1} \operatorname{rank} H\left(i, j, \alpha_{i}\right)-\sum_{i+j=\tau_{i 0}} \operatorname{rank} H\left(i, j, \alpha_{i}\right)\right) \\
& =\underset{\tau_{0} \in \mathbb{N}}{\operatorname{Min}}\left(\sum_{i+j=\tau_{0}+1} \operatorname{rank} H\left(i, j, \alpha_{i}\right)-\sum_{i+j=\tau_{0}} \operatorname{rank} H\left(i, j, \alpha_{i}\right)\right)
\end{aligned}
$$

with $\alpha_{i} \in \mathbf{C}^{q+q^{\prime}}$ having the ith component distinct from unity and the remaining ones being unity; $\mathbf{h}^{\mathbf{i} 0}$ is an associate $\left(q+q^{\prime}\right)$-tuple of delays in $\mathbb{R}_{+}^{q+q^{\prime}}$ with only the ith component being nonzero and $A_{i}=0$, with the remaining ones being zero and $\tau_{0}:=\operatorname{Max}\left(\tau_{i 0}: i \in \overline{q+q^{\prime}}\right)$. Then, the order for any minimal realization independent of the delays is

$$
n=n(\mathbf{h})=n\left(\mathbf{h}^{\mathbf{i} 0}\right)=n_{0}=\tau_{0}=\operatorname{Max}_{\tau\left(\geq \tau_{0}\right) \in \mathbb{N}}\left(\underset{z \in S_{C_{\tau}}(\mathbf{h}) \cup S_{O_{\tau}}(\mathbf{h})}{\operatorname{gen} \operatorname{rank}} H(i+\ell-1, \tau+\ell-i, z)\right) \quad \forall \mathbf{h} \in \mathbb{R}_{+}^{q+q^{\prime}},
$$

and all the matrices defining the state-space realization (2.1)-(2.2) are independent of the delays. 
Proof. Equation (4.4) of property (i) follows directly by using a close reasoning to that in Theorem 3.7(ii) since $n \in \mathbb{N}$ has to exist such that

$$
\begin{aligned}
\operatorname{Max}_{\tau \in \mathbb{N}}\left(\underset{z \in S_{C_{\tau}}(\mathbf{h}) \cup S_{O_{\tau}}(\mathbf{h})}{\mu}\left(H^{\tau}(z)\right)\right) & =\underset{\tau \geq n+i-1, i \geq n, z \in \mathbf{C}^{q+q^{\prime}}}{\operatorname{gen~rank}}\left[H_{G}(z)\right] \\
& =\underset{\tau \geq n+i-1, i \geq n, z \in \mathbf{C}^{q+q^{\prime}}}{\operatorname{gen~rank}}[H(i, \tau+1-i, z)], \\
\underset{\tau \geq n, z \in \mathbf{C}^{q+q^{\prime}}}{\operatorname{gen~} \operatorname{rank}}\left[C_{\tau}\left(A\left(z^{I}\right), B\left(z^{E}\right)\right)\right] & =\underset{\tau \geq n, z \in S_{C_{n}}(\mathbf{h}) \cup S_{O_{n}}(\mathbf{h})}{\operatorname{rank}}\left[C_{\tau}\left(A\left(z^{I}\right), B\left(z^{E}\right)\right)\right] \\
& =n \quad \forall \tau(\geq n) \in \mathbb{N} .
\end{aligned}
$$

Equation (4.4) is identical to (4.2) from the definition of McMillan degrees of minimal realizations, namely, of partial minimal realizations $[4,7]$ and transfer matrices and the fact that the generic ranks of infinite block Hankel matrices $[4,5]$ are constant and equalize that of those exceeding appropriate sizes under certain thresholds. Equation (4.3) equalizes (4.2) and (4.4) by extending a parallel result given in [4] for real block Hankel matrices describing the realization problem of the delay-free case (i.e., $H(i, j)$ does not depend on a multidimensional complex tuple $z$ ) and by the fact that this rank does not increase for $\tau \in \mathbb{N}$ exceeding a certain finite minimum threshold $\tau_{0} \in \mathbb{N}$. Property (i) has been proved. Property (ii) follows in the same way by using similar considerations for any given $\tau \in \mathbb{N}$. To prove property (iii), first note that $\mathbf{h}^{\mathrm{i} 0} \in \mathbb{R}_{+}^{q+q^{\prime}}$ for $i \in \overline{q+q^{\prime}}$ defines each particular delay-free parameterization of (2.1)-(2.2) with some zero delays and the remaining ones being infiniy (or, equivalently, with their associated matrices of dynamics $A_{(\cdot)}$ being null) as follows:

$$
\dot{z}_{i}(t)=\left(\sum_{j=0}^{q} A_{j} i_{A}(q, i, j)\right) z_{i}(t)+\sum_{j=0}^{q^{\prime}}\left(B_{j} i_{B}\left(q, q^{\prime}, i, j\right)\right) u(t) .
$$

$i \in \overline{q+q^{\prime}}$ under initial conditions $z(t)=z_{0}=\varphi(0)$, where the binary indicators

$$
\begin{aligned}
i_{A}(q, i, j)=1 & \text { if } i, j(\neq i) \in \bar{q}, i \in \overline{q+q^{\prime}}, \\
i_{A}(q, i)=0 & \text { if } i, j(=i) \in \bar{q}, \\
i_{B}\left(q, q^{\prime}, i, j\right)=1 & \text { if } i(\neq j) \in \overline{q+q^{\prime}} / \bar{q}, i \in \bar{q}, \\
i_{B}\left(q, q^{\prime}, i, j\right)=0 & \text { if } i, j(=i) \in \overline{q+q^{\prime}} / \bar{q}
\end{aligned}
$$

have been used for notational simplicity, since $\mathbf{h}^{00}=(0,0, \ldots, 0)$ and $\mathbf{h}^{\mathbf{i} 0}$ is defined with components $h_{j}=0$, for all $j(\neq i) \in \overline{q+q^{\prime}}$, and $A_{i}=0$, or $h_{i}=\infty$ if $i \in \bar{q}$ and $B_{i}=0$ or $h_{q+i}=h_{i}^{\prime}=\infty$ if $i \in \overline{q+q^{\prime}} / \bar{q}$. All the delay-free parameterizations (4.10)-(4.11) of (2.1)-(2.2) have a minimal state-space realization of identical dimension $n=n_{0}$ from (4.7). Using (3.11), now independent of the complex indeterminate $z$, for each of the delay-free state-space realizations, Lemma 3.6(i), and the fact that all the above delay-free realizations are spectrally controllable and observable, one can construct $q+q^{\prime}$ algebraic matrix equations to calculate the matrices $\widehat{A}_{i}=\sum_{j(\neq i)=0}^{q} A_{j}, \widehat{B}_{\ell}=\sum_{j(\neq \ell)=0}^{q} B_{j}, i \in / \bar{q} \cup\{0\}, \ell \in \bar{q}^{\prime} \cup\{0\}$, from which $A_{i}, i \in \bar{q} \cup\{0\} ;$ $B_{j}, j \in \bar{q}^{\prime} \cup\{0\}$ can be calculated uniquely for some matrix $C$ such that (3.11) holds with $D=H_{0}$. Since all the matrices of parameters of (2.1)-(2.2) may be calculated, then the minimal order is identical, independent of the delays, so that (4.8) holds. 
It is of interest to provide some result concerning the case when controllability and observability are maintained, and then the order of the minimal realization is not modified, under some parametrical and delay disturbances. In what follows, parametrical perturbations consisting of matrix scaling and constant perturbations of all delays are discussed.

Theorem 4.2. Consider the transfer matrix

$$
\widehat{G}\left(s, \delta, \rho_{1}, \rho_{2}, \lambda, \tilde{h}\right)=\rho_{1} C\left(s I_{n}-\delta \sum_{i=0}^{q} A_{i} e^{-h_{i} s} e^{-\tilde{h} s}\right)^{-1}\left(\sum_{i=0}^{q^{\prime}} \rho_{2} B_{i} e^{-h_{i} s} e^{-\tilde{h}^{\prime} s}\right)+\lambda D
$$

parameterized in the sextuple of real scalars $\mathbf{p}:=\left(\delta, \rho_{1}, \rho_{2}, \lambda, \tilde{h}, \tilde{h}^{\prime}\right)$ which models a perturbation of a nominal transfer matrix

$$
\widehat{G}\left(s, \delta, \rho_{1}, \rho_{2}, \lambda, \tilde{h}\right)=C\left(s I_{n}-\sum_{i=0}^{q} A_{i} e^{-h_{i} s}\right)^{-1}\left(\sum_{i=0}^{q^{\prime}} B_{i} e^{-h_{i} s}\right)+D
$$

parameterized by $\mathbf{p}_{0}:=(1,1,1,1,0,0)$, and assume that the denominator quasipolynomial and all the numerator quasipolynomials of (4.12) possess principal terms [8]. Assume that (2.1)-(2.2) is a minimal realization of (minimal) order $n$ of (4.13). Then, a minimal realization of the same order $n$ of (4.12) is given by (2.1)-(2.2) with the parametrical changes $C \rightarrow \rho_{1} C, A_{i} \rightarrow \delta A_{i}(i \in \bar{q} \cup\{0\}), B_{i} \rightarrow \rho_{2} A_{i}$, $D \rightarrow \lambda D$ and delay changes $h_{i} \rightarrow h_{i}+\widetilde{h}, h_{j}^{\prime} \rightarrow h_{j}^{\prime}+\widetilde{h}^{\prime}\left(i \in \bar{q} \cup\{0\}, j \in \bar{q}^{\prime} \cup\{0\}\right)$ for any finite delay perturbations $\tilde{h}$ and $\tilde{h}^{\prime}$ and for any real $\lambda$ if and only if $\rho_{1} \rho_{2} \delta \neq 0$.

Proof. The block Hankel $(i, j)$-matrix associated with the p-parameterization is related to that associated with the $\mathbf{p}_{0}$-parameterization by

$$
\begin{aligned}
H_{\widehat{G}}(i, j, \mathbf{p})= & \operatorname{Block} \operatorname{Diag}\left(\gamma(s)^{(i-1) / 2}, \gamma(s)^{(i-1) / 2}, \ldots, \gamma(s)^{(i-1) / 2}\right) H_{\widehat{G}}\left(i, j, \mathbf{p}_{0}\right) \\
& \times \operatorname{Block} \operatorname{Diag}\left(\gamma(s)^{(i-1) / 2}, \gamma(s)^{(i-1) / 2}, \ldots, \gamma(s)^{(i-1) / 2}\right) \\
& \times \operatorname{Block} \operatorname{Diag}(\eta(s), \eta(s), \underbrace{j-i+1}, \eta(s))
\end{aligned}
$$

and $H_{0}=\lambda D$, where $\gamma(s, \tilde{h})=\delta e^{-\tilde{h} s}$ and $\eta\left(s, \tilde{h}^{\prime}\right)=\rho_{1} \rho_{2} e^{-\tilde{h}^{\prime} s}$. Since the numerator and denominator quasipolynomials have principal terms, they do not have unstable zeros at infinity, that is, zeros with $\operatorname{Re} s \rightarrow \infty$. Then,

$$
n=\operatorname{Max}_{i, j \in \mathbb{N}}\left(\operatorname{gen} \operatorname{rank} H_{\widehat{G}}\left(i, j, \mathbf{p}_{0}\right)\right)=\operatorname{Max}_{i, j \in \mathbb{N}}\left(\mu\left(H_{\widehat{G}}\left(i, j, \mathbf{p}_{0}\right)\right)\right)=\underset{i, j \in \mathbb{N}}{\operatorname{Max}}\left(\mu\left(H_{\widehat{G}}(i, j, \mathbf{p})\right)\right)
$$

if and only if $\delta \rho_{1} \rho_{2} \neq 0 \Leftrightarrow \gamma(s, \tilde{h}) \eta\left(s, \tilde{h}^{\prime}\right) \neq 0$ for all complex indeterminate $s$ and any finite delay disturbances $\widetilde{h}$ and $\tilde{h}^{\prime}$.

The above result establishes that scalar nonzero scaling of the matrices which parameterize (2.1)-(2.2) preserves the spectral controllability/observability, and then the degree of any minimal realization for any finite constant change of the internal delays and a finite constant change of the external point delays. For these purposes, it is assumed with no loss of generality that the system transfer function is defined by numerator and denominator quasipolynomials 
which possess principal terms.It has been also proved that those properties hold by adding any zero or nonzero interconnection constant matrix to a strictly proper transfer matrix whose nominal state-space realization possesses them. An immediate consequent robustness result for minimal state-space realizations of very easy testing and interpretation applicable to the delay-free single-input single-output case is as follows.

Corollary 4.3. Consider the following three transfer functions:

$$
\begin{aligned}
\widehat{G}_{p}(s) & =\widehat{G}(s) \frac{\tilde{n}(s)}{\tilde{d}(s)} \\
\widehat{G}(s) & =\left(c^{T}\left(s I_{n}-\delta A_{0}\right)^{-1} \rho b_{0}+\lambda d_{0}\right), \\
\widehat{G}_{0}(s) & =\left(c^{T}\left(s I_{n}-A_{0}\right)^{-1} b_{0}+d_{0}\right),
\end{aligned}
$$

where $\widetilde{n}(s)$ and $\widetilde{d}(s)$ are polynomials of respective degrees $\widetilde{n}_{n}(s)=\operatorname{deg}(\widetilde{n}(s))$ and $\widetilde{n}_{d}(s)=\operatorname{deg}(\widetilde{d}(s))$ which satisfy the degree constraint $\tilde{n}_{d}(s) \geq \tilde{n}_{n}(s)+m-n$ with

$$
n=\operatorname{deg}\left(s I_{n}-\delta A_{0}\right), \quad m=\left\{\begin{array}{ll}
n & \text { if } \lambda d_{0} \neq 0, \\
m^{\prime}<n & \text { if } \lambda d_{0}=0,
\end{array} \quad m^{\prime}=\operatorname{deg}\left(c^{T} \operatorname{Adj}\left(s I_{n}-\delta A_{0}\right) \rho b_{0}\right) .\right.
$$

Then, the following properties hold.

(i) The state-space realization $\left(c^{T}, A_{0}, b_{0}, d_{0}\right)$ of $\widehat{G}_{0}(s)$ is both controllable and observable, and then minimal of order $n$, if and only if the associate Hankel matrix satisfies rank $H_{\widehat{G}_{0}}=$ $\operatorname{Max}_{i, j \in \mathbb{N}}\left(\operatorname{rank} H_{\widehat{G}_{0}}(i, j)\right)=\operatorname{rank} H_{\widehat{G}_{0}, j \geq i+n-1 ; i, j \in \mathbb{N}}(i, j)=n_{0}$. Then, $\widehat{G}_{0}(s)$ is proper (strictly proper if $\left.d_{0} \neq 0\right)$ and zero-pole cancellation-free, and $A_{0}$ is of order $n_{0}$.

(ii) Assume that the state-space realization $\left(c^{T}, A_{0}, b_{0}, d_{0}\right)$ of $\widehat{G}_{0}(s)$ is controllable and observable of minimal order $n_{0}$. Then, the state-space realization $\left(c^{T}, \delta A_{0}, \rho b_{0}, \lambda d_{0}\right)$ of $\widehat{G}(s)$ is both controllable and observable, and then minimal of order $n=n_{0}$, if and only if $\delta \rho \neq 0$. Then, $\widehat{G}(s)$ is proper (strictly proper if $\lambda d_{0} \neq 0$ ) and zero-pole cancellation-free, and $A$ is of order $n=n_{0}$. As a result,

$$
\operatorname{rank} H_{\widehat{G}}=\underset{i, j \in \mathbb{N}}{\operatorname{Max}}\left(\operatorname{rank} H_{\widehat{G}}(i, j)\right)=\underset{j \geq i+n-1 ; i, j \in \mathbb{N}}{\operatorname{rank}} H_{\widehat{\widehat{N}}}(i, j)=n .
$$

(iii) $\widehat{G}_{p}(s)$ is state-space realizable and strictly proper if and only if $\widetilde{n}_{d}(s)>\widetilde{n}_{n}(s)+m-n$. Assume that $\widehat{G}_{0}(s)$ is zero-pole cancellation-free of order $n_{0}$. Then, the following hold.

(1) $\widehat{G}_{p}(s)=n_{\widehat{G}}(s) / d_{\widehat{G}}(s)$ is cancellation-free if and only if $\rho \delta \neq 0$ and the three pairs of polynomials $\left(n_{\widehat{G}}(s), \tilde{d}(s)\right),\left(d_{\widehat{G}}(s), \tilde{n}(s)\right)$, and $(\tilde{d}(s), \tilde{n}(s))$ are each coprime, where $d_{\widehat{G}}(s)=\left(s I_{n}-\delta A_{0}\right)$ and $n_{\widehat{G}}(s)=c^{T} \operatorname{Adj}\left(s I_{n}-\delta A_{0}\right) \rho b_{0}+\lambda d_{0} d_{\widehat{G}}(s)$ are the denominator and numerator polynomials of $\widehat{G}_{p}(s)$. As a result, $\widehat{G}_{p}(s)$ has a minimal realization (then, controllable and observable) of order $\widehat{n}=n\left(=n_{0}\right)+\widetilde{n}_{d}$.

(2) Assume that $\widehat{G}_{0}(s)$ is zero-pole cancellation-free of order $n_{0}$ and $\rho \delta \neq 0$. Assume also that the polynomial pairs $\left(n_{\widehat{G}}(s), \tilde{d}(s)\right),\left(d_{\widehat{G}}(s), \widetilde{n}(s)\right)$ are both coprime. Then, $\widehat{G}_{p}(s)$ has zero-pole cancellation(s) at the common factors of the pair $(\tilde{d}(s), \widetilde{n}(s))$, if any. A minimal 
realization of $\widehat{G}_{p}(s)$ has order $\widehat{n}=n_{0}+\widetilde{n}_{d}-\widetilde{n}_{d^{\prime}}^{\prime}$, which satisfies $n_{0}+\widetilde{n}_{d} \geq \widehat{n} \geq n_{0}$, where $\tilde{n}_{d}^{\prime} \geq 0$ is the degree of the cancellation polynomial, if any. Also,

$$
n_{0}+\tilde{n}_{d} \geq \operatorname{rank} H_{\widehat{G}_{p}}=\underset{i, j \in \mathbb{N}}{\operatorname{Max}}\left(\operatorname{rank} H_{\widehat{G}_{p}}(i, j)\right)=\underset{\substack{j \geq i+\hat{n}-1 ; i, j \in \mathbb{N} \\ \operatorname{rank}}}{ } H_{\widehat{G}_{p}}(i, j)=\widehat{n} \geq n_{0} .
$$

\section{Outline of proof}

The proof follows directly from Theorem 4.2 by noting from its definitions of the various transfer functions that $\widehat{G}(s)=\widehat{G}_{0}(s)$ if $\rho=1$ and $\lambda=0$, and $\widehat{G}_{p}(s)=\widehat{G}(s)$ if $\tilde{n}(s)=\tilde{d}(s)$. As a result, $\widehat{G}_{p}(s)$ is zero-pole cancellation-free if the polynomials $\widetilde{n}(s)$ and $\widetilde{d}(s)$ are nonzero real scalars provided that $\widehat{G}_{0}(s)$ is also cancellation-free. The transfer function $\widehat{G}_{p}(s)$ is also cancellationfree if $\widehat{G}_{0}(s)$ and $\tilde{n}(s) / \widetilde{d}(s)$ are both cancellation-free and, furthermore, $\left(\widetilde{n}(s), \operatorname{Det}\left(s I_{n}-A_{0}\right)\right)$ and $\left(\tilde{d}(s), c^{T} \operatorname{Adj}\left(s I_{n}-A_{0}\right) b_{0}+d_{0} \operatorname{Det}\left(s I_{n}-A_{0}\right)\right)$ are both coprime pairs of polynomials.

Then, proceed as follows to complete the proof of the various properties.

(1) Remove the delays and consider the single-input single-output case by relating ranks of infinite or partial block Hankel matrices with orders being minimal, then being controllable and observable.

(2) Note those minimal state-spacerealizations which cannot have zero-pole cancellations in their transfer function and vice versa.

(3) If there are cancellations, then the associate realization is never minimal.

The above result can be extended very easily to the multivariable case and to the presence of delays.

\section{Concluding remarks}

This paper addresses the problem of synthesizing minimal realizations and partial minimal realizations of linear time-invariant systems with (in general, incommensurate) multiple constant internal and external point delays. The main body of the formalism of the properties of controllability, observability, minimal realizations, and minimal partial realizations is discussed through a formulation over appropriate rings of polynomials and corresponding (roughly speaking, isomorphic) truncations of formal Laurent expansions of rational transfer matrices. However, the spectral versions of controllability and observability are used to remove spurious conditions which lead to apparent loss of those properties. In this sense, the presented results are stronger than those previous parallel ones derived in a full formalism over rings. Some particular results are also obtained for the single-input single-output case by mutually relating realizations of transfer functions with given basic control and output vectors, inputoutput interconnection gain, and dynamics matrix, but being on the other hand dependent at most on three potentially freely chosen real parameters. The minimal state-space realizations are interpreted in terms of absence of zero-pole cancellation in the transfer function by giving some direct relationships among those parameters. Since one starting point for the analysis is the Hankel matrix, the formalism is appropriate to be applied for obtaining transfer matrices, minimal realizations, and minimal partial realizations collected input-output data. 


\section{Acknowledgments}

The author is very grateful to the Spanish Ministry of Education for its partial support of this work through Project no. DPI 2006-00714. He is also grateful to the reviewers for their useful comments.

\section{References}

[1] R. E. Kalman, "Mathematical description of linear dynamical systems," SIAM Journal on Control and Optimization, vol. 1, no. 2, pp. 152-192, 1963.

[2] R. E. Kalman, "On partial realizations, transfer functions, and canonical forms," Acta Polytechnica Scandinavica, no. 31, pp. 9-32, 1979.

[3] I. Gohberg, M. A. Kaashoek, and L. Lerer, "On minimality in the partial realization problem," Systems E Control Letters, vol. 9, no. 2, pp. 97-104, 1987.

[4] D. Hinrichsen, W. Manthey, and U. Helmke, "Minimal partial realization by descriptor systems," Linear Algebra and Its Applications, vol. 326, no. 1-3, pp. 45-84, 2001.

[5] I. S. Iohvidov, Hankel and Toeplitz Matrices and Forms: Algebraic Theory, Birkhäuser, Boston, Mass, USA, 1982.

[6] W. Manthey, D. Hinrichsen, and U. Helmke, "On Fischer-Frobenius transformations and the structure of rectangular block Hankel matrices," Linear and Multilinear Algebra, vol. 41, no. 3, pp. 255-288, 1996.

[7] W. Manthey and U. Helmke, "Connectivity properties of spaces of partial realizations," Systems $\mathcal{E}$ Control Letters, vol. 43, no. 3, pp. 225-238, 2001.

[8] S.-I. Niculescu, Delay Effects on Stability. A Robust Control Approach, vol. 269 of Lecture Notes in Control and Information Sciences, Springer, London, 2001.

[9] M. De la Sen, "On impulsive time-varying systems with unbounded time-varying point delays: stability and compactness of the relevant operators mapping the input space into the state and output spaces," The Rocky Mountain Journal of Mathematics, vol. 37, no. 1, pp. 79-129, 2007.

[10] J. Wang, F. Meng, and S. Liu, "Integral average method for oscillation of second order partial differential equations with delays," Applied Mathematics and Computation, vol. 187, no. 2, pp. 815-823, 2007.

[11] Y. G. Sun, L. Wang, and G. Xie, "Delay-dependent robust stability and $H_{\infty}$ control for uncertain discrete-time switched systems with mode-dependent time delays," Applied Mathematics and Computation, vol. 187, no. 2, pp. 1228-1237, 2007.

[12] J. Jiao, X. Meng, and L. Chen, "A stage-structured Holling mass defence predator-prey model with impulsive perturbations on predators," Applied Mathematics and Computation, vol. 189, no. 2, pp. 1448 $1458,2007$.

[13] X. Liao and S. Guo, "Delay-dependent asymptotic stability of Cohen-Grossberg models with multiple time-varying delays," Discrete Dynamics in Nature and Society, vol. 2007, Article ID 28960, 17 pages, 2007.

[14] Q. Zhang, X. Wei, and J. Xu, "On global exponential stability of discrete-time Hopfield neural networks with variable delays," Discrete Dynamics in Nature and Society, vol. 2007, Article ID 67675, 9 pages, 2007.

[15] L. Cai, X. Li, X. Song, and J. Yu, "Permanence and stability of an age-structured prey-predator system with delays," Discrete Dynamics in Nature and Society, vol. 2007, Article ID 54861, 15 pages, 2007.

[16] M. De la Sen, "On positivity of singular regular linear time-delay time-invariant systems subject to multiple internal and external incommensurate point delays," Applied Mathematics and Computation, vol. 190, no. 1, pp. 382-401, 2007.

[17] M. De la Sen, "About $K$-positivity properties of time-invariant linear systems subject to point delays," Journal of Inequalities and Applications, vol. 2007, Article ID 25872, 28 pages, 2007.

[18] X. Ding and H. Su, "Dynamics of a discretization physiological control system," Discrete Dynamics in Nature and Society, vol. 2007, Article ID 51406, 16 pages, 2007.

[19] W. Wang, J. Shen, and J. J. Nieto, "Permanence and periodic solution of predator-prey system with Holling type functional response and impulses," Discrete Dynamics in Nature and Society, vol. 2007, Article ID 81756, 15 pages, 2007.

[20] S. Hilout, M. Boutat, and J. Grilhé, "Plastic deformation instabilities: lambert solutions of MeckingLücke equation with delay," Mathematical Problems in Engineering, vol. 2007, Article ID 45951, 13 pages, 2007. 
[21] M. De la Sen, "Oscillatory behavior in linear difference equations under unmodeled dynamics and parametrical errors," Mathematical Problems in Engineering, vol. 2007, Article ID 25692, 18 pages, 2007.

[22] N. Bradul and L. Shaikhet, "Stability of the positive point of equilibrium of Nicholson's blowflies equation with stochastic perturbations: numerical analysis," Discrete Dynamics in Nature and Society, vol. 2007, Article ID 92959, 25 pages, 2007.

[23] L. Bakule, J. Rodellar, and J. M. Rossell, "Controllability-observability of expanded composite systems," Linear Algebra and Its Applications, vol. 332-334, pp. 381-400, 2001.

[24] L. Bakule, J. Rodellar, J. M. Rossell, and Pere Rubió, "Preservation of controllability-observability in expanded systems," IEEE Transactions on Automatic Control, vol. 46, no. 7, pp. 1155-1162, 2001.

[25] S. Chaib, D. Boutat, A. Benali, and J. P. Barbot, "Observability of the discrete state for dynamical piecewise hybrid systems," Nonlinear Analysis: Theory, Methods E Applications, vol. 63, no. 3, pp. 423438, 2005.

[26] C. Coll, M. J. Fullana, and E. Sánchez, "Reachability and observability indices of a discrete-time periodic descriptor system," Applied Mathematics and Computation, vol. 153, no. 2, pp. 485-496, 2004.

[27] M. De la Sen and N. S. Luo, "Design of linear observers for a class of linear hybrid systems," International Journal of Systems Science, vol. 31, no. 9, pp. 1077-1090, 2000

[28] M. Heyouni and K. Jbilou, "Matrix Krylov subspace methods for large scale model reduction problems," Applied Mathematics and Computation, vol. 181, no. 2, pp. 1215-1228, 2006.

[29] V. M. Marchenko and O. N. Poddubnaya, "Solution representations and relative controllability of linear differential algebraic systems with several delays," Doklady Mathematics, vol. 72, no. 2, pp. 824 828, 2005.

[30] V. M. Marchenko, O. N. Poddubnaya, and Z. Zaczkiewicz, “On the observability of linear differentialalgebraic systems with delays," IEEE Transactions on Automatic Control, vol. 51, no. 8, pp. 1387-1392, 2006.

[31] V. M. Marchenko and O. N. Poddubnaya, "Representation of solutions of hybrid differentialdifference systems," Differential Equations, vol. 42, no. 6, pp. 789-804, 2006.

[32] H. Saadaoui, N. Manamanni, M. Djemaï, J. P. Barbot, and T. Floquet, “Exact differentiation and sliding mode observers for switched Lagrangian systems," Nonlinear Analysis: Theory, Methods E Applications, vol. 65, no. 5, pp. 1050-1069, 2006.

[33] M. De la Sen and N. Luo, "On the uniform exponential stability of a wide class of linear time-delay systems," Journal of Mathematical Analysis and Applications, vol. 289, no. 2, pp. 456-476, 2004.

[34] M. De la Sen, "Stability of impulsive time-varying systems and compactness of the operators mapping the input space into the state and output spaces," Journal of Mathematical Analysis and Applications, vol. 321, no. 2, pp. 621-650, 2006.

[35] A. W. Olbrot, "Observability and observers for a class of linear systems with delays," IEEE Transactions on Automatic Control, vol. 26, no. 2, pp. 513-517, 1981.

[36] E. B. Lee and A. W. Olbrot, "On reachability over polynomial rings and a related genericity problem," International Journal of Systems Science, vol. 13, no. 1, pp. 109-113, 1982.

[37] E. B. Lee and A. W. Olbrot, "Observability and related structural results for linear hereditary systems," International Journal of Control, vol. 34, no. 6, pp. 1061-1078, 1981.

[38] M. De la Sen, "On the stabilizability, controllability and observability of linear hereditary systems with distributed commensurate delays," International Journal of Systems Science, vol. 24, no. 1, pp. 33 $51,1993$.

[39] T. Kailath, Linear Systems, Prentice-Hall Information and System Sciences Series, Prentice-Hall, Englewood Cliffs, NJ, USA, 1980. 


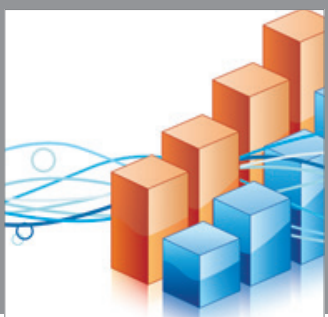

Advances in

Operations Research

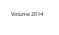

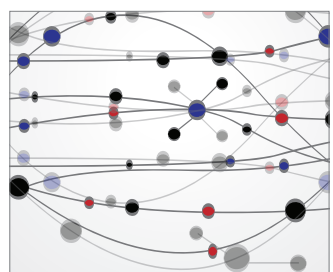

\section{The Scientific} World Journal
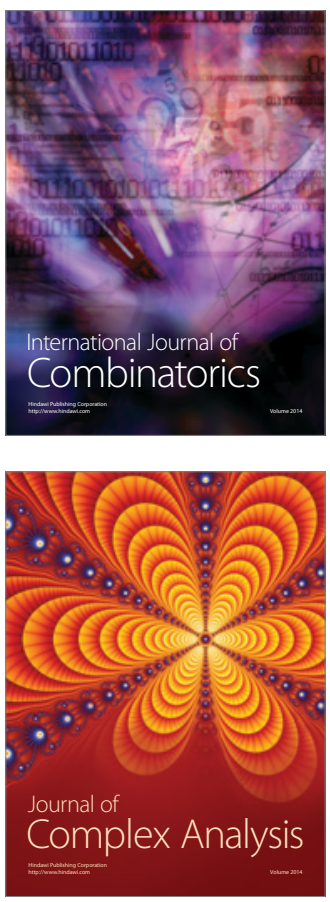

International Journal of

Mathematics and

Mathematical

Sciences
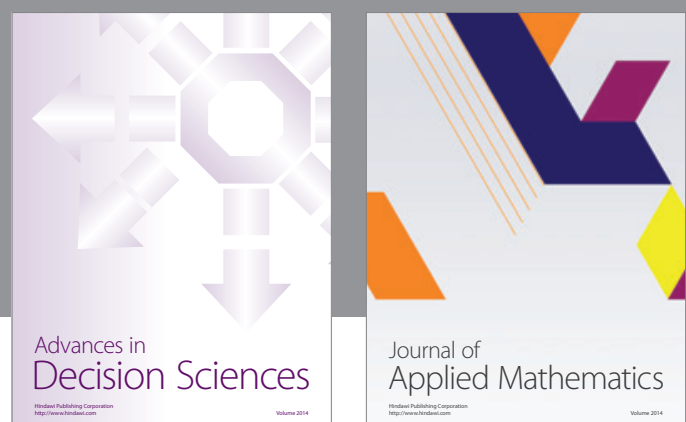

Journal of

Applied Mathematics
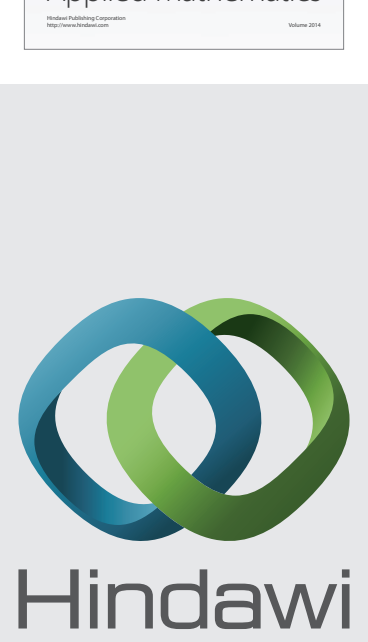

Submit your manuscripts at http://www.hindawi.com
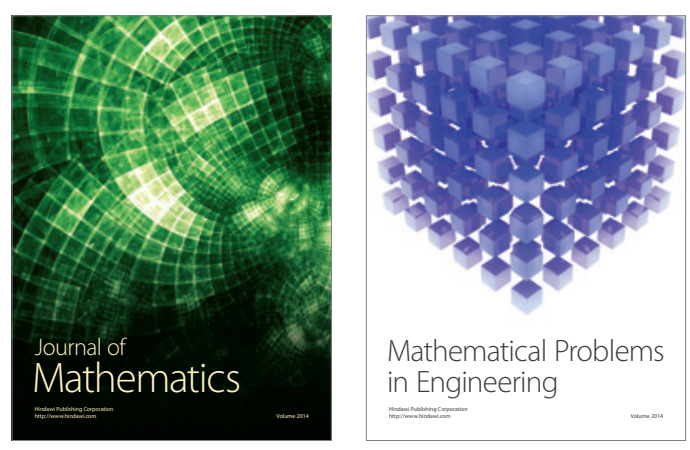

Mathematical Problems in Engineering
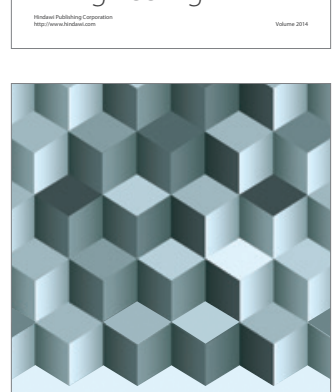

Journal of

Function Spaces
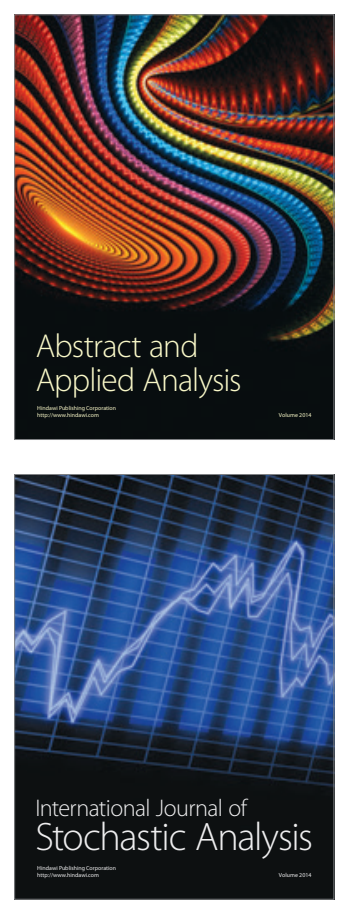

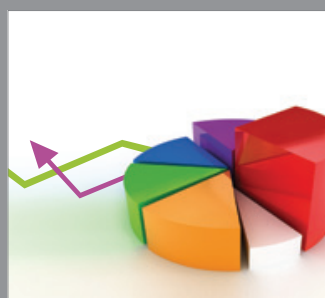

ournal of

Probability and Statistics

Promensencen
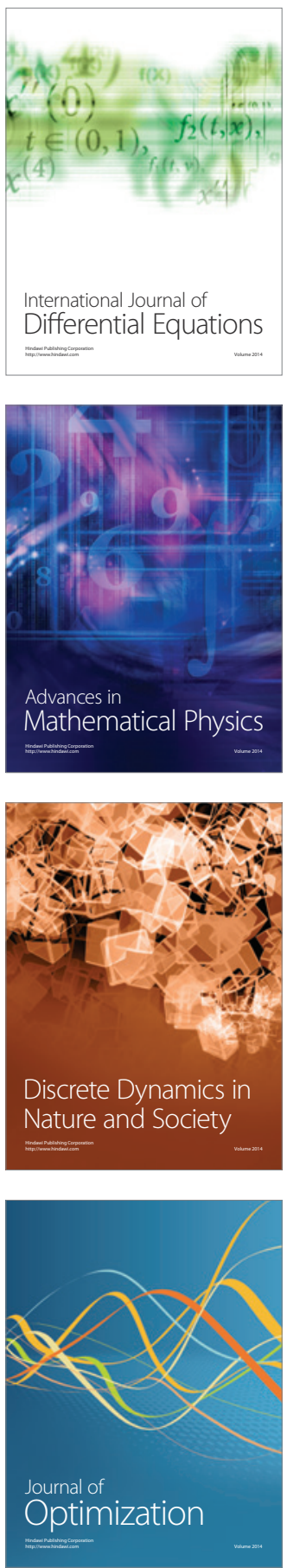